\begin{tabular}{|c|c|}
\hline Title & Fluorescence lifetime images of green fluorescent protein in HeLa cells during TNF- $\alpha$ induced apoptosis \\
\hline Author(s) & Ito, Toshiyuki; Oshita, Shugo; Nakabayashi, Takakazu; Sun, Fan; Kinjo, Masataka; Ohta, Nobuhiro \\
\hline Citation & $\begin{array}{l}\text { Photochemical \& Photobiological Sciences, 8(6), 763-767 } \\
\text { https://doi.org/10.1039/10902341k }\end{array}$ \\
\hline Issue Date & $2009-06$ \\
\hline Doc URL & http:/hdl.handle.net/2115/43088 \\
\hline Rights & Photochem. Photobiol. Sci., 2009, 8, 763-767- Reproduced by permission of The Royal Society of Chemistry (RSC) \\
\hline Type & article (author version) \\
\hline File Information & PPS8-6_p763-767.pdf \\
\hline
\end{tabular}

Instructions for use 


\section{Fluorescence lifetime images of green fluorescent protein in HeLa cells} during TNF- $\alpha$ induced apoptosis

Toshiyuki Ito, ${ }^{a}$ Shugo Oshita, ${ }^{a}$ Takakazu Nakabayashi, ${ }^{a}$ Fan Sun, ${ }^{b}$ Masataka Kinjo ${ }^{b}$ and Nobuhiro Ohta* ${ }^{a}$

${ }^{a}$ Research Institute for Electronic Science, Hokkaido University, Sapporo 001-0020, Japan.

${ }^{b}$ Faculty of Advanced Life Science, Hokkaido University, Sapporo 001-0021, Japan.

*Corresponding should be addressed, Tel: +81 11706 9410; Fax: +81 11706 9406;

E-mail: nohta@es.hokudai.ac.jp 


\begin{abstract}
Fluorescence lifetime images of HeLa cells expressing enhanced green fluorescent protein (EGFP) have been measured, as apoptosis is induced by tumor necrosis factor- $\alpha$ (TNF- $\alpha$ ) in combination with cycloheximide. The fluorescence lifetimes of EGFP is found to decrease after the induction of apoptosis, indicating that the change in environment occurs around the chromophore of EGFP with the apoptosis process. The fluorescence lifetime imaging technique can be used to perform in vivo observation of cell death processes. Fluorescence lifetime measurements are useful to examine the induction of the apoptosis process, even when a morphological change of each cell cannot be observed because of a low spatial resolution.
\end{abstract}

\title{
Keywords
}

Fluorescence lifetime imaging (FLIM), Apoptosis, HeLa cell, Enhanced green fluorescent protein (EGFP), Tumor necrosis factor- $\alpha$ (TNF- $\alpha$ ), Cycloheximide 


\section{Introduction}

Cell death plays a crucially important part in animal and plant development. ${ }^{1}$ One of the cell death ways is that cells activate an intracellular death program and kill themselves in a controlled way. This process is known as apoptosis (or programmed cell death). Apoptosis is characterized by morphological changes such as cell shrinkage, chromatin condensation, blebbing and formation of apoptotic bodies. ${ }^{2}$ The caspase family has been considered as central, indispensable executioners of apoptosis and responsible for major parts of morphological changes. ${ }^{3}$

GFP derivatives including enhanced green fluorescent protein (EGFP) ${ }^{4,5}$ can be also used as a tool to measure apoptosis. ${ }^{6-11}$ In most of the works, GFP and its variants were used as a marker for detection of the morphological changes of apoptotic cells, and the intensity distribution was measured during the apoptosis. The fluorescence intensity of GFP is observed to decrease with apoptosis in some cases; ${ }^{10,11}$ however, cell death processes are difficult to be analyzed quantitatively using fluorescence intensity measurements because of the ambiguity of absolute fluorescence intensity. To overcome these problems, fluorescence lifetime imaging (FLIM) is applicable, ${ }^{12-19}$ and small changes in cellular environment around fluorescent chromophores can be discussed using FLIM. Note that fluorescence lifetime is an inherent property of a 
chromophore, and is independent of the excitation light intensity, excitation wavelength or other experimental factors that limit intensity-based steady-state measurements. Since fluorescence lifetime depends on the local environment that affects the non-radiative rate of the chromophore, FLIM can become a powerful tool for quantitative imaging of cellular microenvironments. In fact, FLIM has been used to examine $\mathrm{pH}$, calcium ion concentration, oxygen concentration, and protein interactions in living cells. ${ }^{12-19}$

In the present study, we have measured the fluorescence lifetime images of HeLa cells expressing EGFP in order to follow and elucidate single-cell dynamics in each cell, when the apoptosis inducer, i.e., TNF- $\alpha$ and cycloheximide are added. ${ }^{3}$ It is shown that the FLIM technique performs in vivo observation of change in environment of the chromophore of EGFP during the apoptosis process.

\section{Materials and Methods}

HeLa cells were cultured in Dulbecco’s modified Eagle’s medium (DMEM, D5796, Sigma) supplemented with $2 \times 10^{5} \mathrm{U} \mathrm{dm}^{-3}$ penicillin G, $200 \mathrm{mg} \mathrm{dm}^{-3}$ streptomycin sulfate, and $10 \%$ fetal bovine serum. Cells were incubated in humidified air with 5\% $\mathrm{CO}_{2}$ at $37^{\circ} \mathrm{C}$. HeLa cells were transfected overnight with $0.5 \mu \mathrm{g} / \mathrm{ml}$ of plasmid DNA 
encoding the EGFP using Optifect Transfection Reagent (12579-017, Invitrogen) in Lab-Tek 8-well chambered coverglass (155411, Nalge Nunc International). Just before the imaging measurements, the medium in the chambered coverglass was replaced with $0.4 \mathrm{ml}$ phenol red-free Opti-MEM I reduced-serum medium (11058-021, Invitrogen) containing 250 ng/ml TNF- $\alpha$ (JM-1050-1, Med. \& Biol. Lab.) and $14 \mu \mathrm{g} / \mathrm{ml}$ cycloheximide (CHX, BV-1041-7, Med. \& Biol. Lab.) to induce apoptosis.

The annexin $\mathrm{V}$ assay was carried out using an annexin V-FITC apoptosis kit (Clontech). HeLa cells were induced to undergo apoptosis, and stained and incubated with annexin V-FITC according to the protocol provided every two hours. The annexin V-FITC positive cells were observed by fluorescence microscopy. Necrosis in HeLa cells was induced by staurosporin (STS) under conditions of intracellular ATP depletion. $^{20}$ HeLa cells expressing EGFP were incubated with $2 \mathrm{mM}$ pyruvate and 2.5 $\mu \mathrm{M}$ oligomycin in serum-free DMEM without glucose for 45 minutes and then exposed to $3 \mu \mathrm{M}$ STS to induce cell death.

The method used in the FLIM measurements is based on the time-gated detection of the fluorescence intensity. A full description of the experimental system was described elsewhere. ${ }^{17-19}$ The FLIM measurements were carried out with an inverted confocal laser scanning microscope (Digital Eclipse C1, Nikon). An output from a 
mode-locked Ti:Sapphire laser (Tsunami, Spectra-Physics) pumped by a solid state diode laser (Millennia Xs, Spectra-Physics) was doubled by a BBO crystal in an ultrafast harmonic generator (Model 5-050, Inrad). The excitation light at $405 \mathrm{~nm}$ or $440 \mathrm{~nm}$ with a repetition frequency of $81 \mathrm{MHz}$ was used. The laser beam was introduced into a scanner unit of the confocal microscope via a single mode optical fiber. A neutral density filter was used to attenuate the excitation light intensity. The excitation beam was focused onto a sample with an objective lens (CFI Plan 40×, NA 0.95, Nikon). Fluorescence from the sample was collected with the same objective lens, and introduced into a multimode optical fiber. The confocal pinhole diameter was adjusted to $150 \mu \mathrm{m}$. The collected fluorescence was transmitted into two filters (BA520 and EX510-560, Nikon) for the $440 \mathrm{~nm}$ excitation and one interference filter (VPF-25C-10-50-50850, Sigma Koki) for the $405 \mathrm{~nm}$ excitation, and detected using a high-speed lifetime imaging module (LIMO, Nikon Europe BV). Fluorescence lifetime at each pixel of the image was calculated from the four time-windows signals by assuming a single exponential decay. The time-interval of each window was set to be 2 ns. The image size was $256 \times 256$ pixels. Each image was accumulated by 256 times. The acquisition time was 6.5 min for each image. All the measurements were performed in a stage top incubator (INUG2-NI, Tokai Hit) with $5 \% \mathrm{CO}_{2}$ at $37^{\circ} \mathrm{C}$. 


\section{Results and Discussion}

The FLIM images as well as the fluorescence intensity images of HeLa cells expressing EGFP were measured as a function of time-lapse after adding the TNF- $\alpha / \mathrm{CHX}$, which induces apoptosis. The results obtained by monitoring the fluorescence in the region of 515 - $560 \mathrm{~nm}$ with excitation at $440 \mathrm{~nm}$ are shown in Fig.

1. Fig. 1(a) $(0 \mathrm{~h})$ shows the results just after adding TNF- $\alpha / C H X$. EGFP is distributed in cytosol and also in the nucleus due to the absence of a specific targeting sequence. The nearly uniform distribution of the fluorescence intensity in each cell indicates that EGFP can pass through nuclear pores. Similar to the intensity images, the FLIM images display a mostly uniform distribution throughout the entire cell (see Fig. 1(b) $(0 \mathrm{~h}))$ in the present spatial resolution, and the average fluorescence lifetime ( $\left.\tau_{\mathrm{av}}\right)$ at $0 \mathrm{~h}$ is determined to be $2.32 \mathrm{~ns}$ (Fig. 1(c)), which is in good agreement with the

reported values in cells. ${ }^{21-23}$ Note that $\tau_{\text {av }}$ is taken from the maximum of the histogram of FLIM.

We measured the FLIM images as well as fluorescence intensity images every one hour after TNF- $\alpha / \mathrm{CHX}$ was added. The results obtained with a lapse of 2, 4 or 6 hours are shown in Fig. $1(2,4,6 \mathrm{~h})$. The intensity images show that the apoptosis 
progresses as a passage of time; the cells show the apoptotic cell death morphology, including membrane blebbing and cell shrinkage, with a lapse of 4 or 6 hours. The corresponding lifetime images show that $\tau_{\mathrm{av}}$ decreases with a passage of time, along with the occurrence of the apoptosis. With a lapse of $6 \mathrm{~h}$, for example, $\tau_{\mathrm{av}}$ decreases to 2.27 ns, as shown in Fig. 1, indicating that the microenvironment around the EGFP chromophore is changed by the apoptosis. Besides the decrease of $\tau_{\mathrm{av}}$, the distribution of the lifetime becomes larger as a passage of time, suggesting not only that the apoptotic cells give a shorter lifetime but also that the progress of the apoptosis depends on the cell. In fact, the cells give different time-lapse dependence of the FLIM image from each other: the magnitude of the shift of $\tau_{\mathrm{av}}$ as well as the extent of the broadening of the histogram depends on the cell, as clearly shown in Fig. 2. It should be stressed that the lifetime in each cell is not the same, but some distribution is noticed. Unfortunately, the space resolution in the present experiments is not high enough to discuss the detailed space distribution of the fluorescence lifetime inside the cell.

As mentioned above, the apoptosis induces the change in $\tau_{\text {av }}$ of HeLa cells expressing EGFP. In order to confirm the apoptosis-induced change in lifetime, FLIM was observed in the absence of apoptosis inducer. Fig. 3(a) shows fluorescence intensity images of HeLa cells expressing EGFP observed without apoptosis inducer, i.e., without 
TNF- $\alpha / \mathrm{CHX}$. Fluorescence in the region of 515 - $560 \mathrm{~nm}$ was observed with excitation at $440 \mathrm{~nm}$. The normal morphology was observed even with a passage of long time, i.e., even after $6 \mathrm{~h}$, and the shift of $\tau_{\mathrm{av}}$ was not observed, as shown in Fig. 3(c). It may be worth mentioning that the fluorescence lifetime of EGFP located in the peripheral part of each cell seems to be shorter than that of EGFP located at the inner part of the cell, though the space resolution was not so good in the present experiments, unfortunately.

A great deal of work has been published for photophysical properties of wild-type GFP and its derivatives including EGFP. ${ }^{4,5}$ It has been reported that absorption and fluorescence spectra, and fluorescence quantum yield of GFPs depend on environments such as $\mathrm{pH}^{24,25}$ halide ions, ${ }^{26}$ and photoirradiation. ${ }^{27}$ According to the reports, ${ }^{28,29}$ apoptosis is often associated with acidification of the cytosol. In apoptotic cells, intra-cellular $\mathrm{pH}$ was decreased from 7.2 to 5.7 in U937 cells exposed to TNF- $\alpha{ }^{29}$ Therefore, a possibility must be considered that the decrease of EGFP fluorescence lifetime in apoptotic cells was caused by the cytosolic acidification of the HeLa cells exposed to TNF- $\alpha$. Heikal et al. have reported a remarkable $\mathrm{pH}$ dependence of the fluorescence lifetime of EGFP with excitation of the neutral species of the EGFP chromophore. $^{24}$ If the cytosolic acidification is the reason, therefore, it is expected for 
the excitation of the neutral (protonated) species of the EGFP chromophore that a large decrease occurs following the apoptosis in fluorescence lifetime of EGFP whose magnitude is much larger than the one observed for the excitation of the anionic species. In fact, FLIM with excitation of neutral species of the EGFP chromophore shows a clear $\mathrm{pH}$ dependence of HeLa cells expressing EGFP. ${ }^{18}$ In order to confirm the $\mathrm{pH}$ dependence, we carried out the FLIM measurements with excitation of the neutral species, i.e., with excitation at $405 \mathrm{~nm}$ and detection of the fluorescence at around 510 nm. The results are shown in Fig. 4, where FLIM images as well as intensity images observed in the presence of TNF- $\alpha / \mathrm{CHX}$ are shown as a function of lapse time, together with the histograms of the lifetime for the whole cells at the lapse time of 0 and 8 hours, respectively. As in the case of the $440 \mathrm{~nm}$ excitation, $\tau_{\mathrm{av}}$ becomes shorter as a passage of time, i.e., as the apoptosis occurs, but the magnitude of the shift is similar to the one observed with the $440 \mathrm{~nm}$ excitation. The decrease of the fluorescence lifetime induced by the apoptosis observed for the excitation of the neutral species is similar in extent to the one observed for the excitation of the anionic species. Then, it is concluded that the observed apoptosis-induced change in lifetime does not come from the $\mathrm{pH}$ dependence. The $\tau_{\mathrm{av}}$ value of EGFP with excitation at $405 \mathrm{~nm}$ is observed to be a little larger than that with excitation at $440 \mathrm{~nm}$, which is in agreement with the 
observation in buffer solution with almost the same excitation and fluorescence wavelengths. $^{30}$ This behavior probably arises from the change in the ratio of the fluorescence intensities of the two deprotonated excited species with the excitation wavelength. $^{30}$

The cells that are undergoing apoptosis show multitude of characteristic and tightly regulated morphological and biochemical features. Some of the most typical attributes of apoptosis include dehydration followed by cell shrinkage, loss of mitochondrial transmembrane potential, and release of cytochrome c from mitochondria. DNA fragmentation, shift in cellular redox state, ${ }^{31}$ and changes in concentrations of intracellular ions have also been observed. ${ }^{32}$ The loss of plasma asymmetry is one of the key features of the early stage of apoptosis and the redistribution of phosphatidylserine (PS) to the outer layer of the plasma membrane can be detected with annexin $\mathrm{V}$ that has a strong affinity for PS. As shown in Fig. 4, time-lapse experiments of annexin V-FITC staining of apoptosis suggest that HeLa cells treated to undergo apoptosis mostly exhibit PS externalization within 4 hours in the present experimental condition. This result indicates that the decrease in fluorescence lifetime occurs after PS externalization. After 6 hours from the induction of apoptosis, some of HeLa cells exhibiting apoptotic cell death morphology were stained with propidium 
iodide (PI), indicating the loss of plasma membrane integrity of these cells. However, the decrease in fluorescence lifetime seems not to be observed for HeLa cell expressing EGFP treated to undergo necrosis, although the autofluorescence in cells undergoing necrosis interferes with the detailed analysis of the lifetime of EGFP in the present condition. Further experiments are necessary to clarify the relationship between the loss of plasma membrane integrity and the change in fluorescence lifetime.

Recently, Li et al. have reported the application of FLIM to an apoptosis assay using SYTO13 probe. ${ }^{33}$ SYTO13 binds to DNA in nucleus, and the decrease in fluorescence lifetime was observed in apoptosis process. They explained the result from self-quenching of the fluorescence induced by a decrease in the distance between the dye molecules that arises from the condensation of DNA during apoptosis. The present decrease in the fluorescence lifetime does not arise from self-quenching of EGFP because the observed fluorescence lifetime is independent of the fluorescence intensity.

DNA fragmentation, shift in cellular redox state, or change in concentrations of intracellular ions will induce the environmental change surrounding the GFP chromophore. As a result of the apoptosis, therefore, the electrostatic field surrounding the EGFP chromophore may change. Changing in the local electric field 
produced by the protein matrix surrounding the EGFP chromophore is also expected. ${ }^{19,34}$ Cells undergoing apoptosis often lose the electrical potential that normally exists across the inner membrane of their mitochondria., ${ }^{1,9,29}$ It is suggested that the redox changes occurring during the apoptotic process ${ }^{31}$ are responsible for the decrease of fluorescence lifetime. Then, the decrease of the fluorescence lifetime observed during the apoptosis may be ascribed to a change in electric field surrounding the EGFP chromophore. Further study is necessary to elucidate the mechanism of the apoptosis-induced change in fluorescence lifetime of EGFP in HeLa cells. However, it should be stressed that the change in fluorescence lifetime will be useful to examine the apoptosis process, even when the morphological change cannot be observed by a low spatial resolution.

\section{Conclusion}

Fluorescence lifetime images of HeLa cells expressing EGFP have been measured during the apoptosis which was induced by TNF- $\alpha / \mathrm{CHX}$. As the apoptosis occurred in a cell, a small but clearly discernible decrease of the fluorescence lifetime of the EGFP fluorescence was observed, along with a morphological change, indicating that the apoptosis-induced change in environment occurs around the fluorescent chromophore of 
EGFP. The apoptosis-mediated decrease in the fluorescence lifetime of EGFP may be ascribed to an apoptosis-induced change in the local electrostatic field surrounding the EGFP chromophore, which probably enhances the nonradiative decay rate at the emitting state of the EGFP chromophore. FLIM of EGFP can be used to perform in vivo observation of cell death processes. 


\section{Note and references}

1. B. Alberts, A. Johnson, J. Lewis, M. Raff, K. Roberts and P. Walter, Molecular Biology of the Cell, Garland Science, New York, fifth edn., 2008.

2. R. T. Allen, W. J. Hunter and D. K. Agrawal, Morphological and biochemical characterization and analysis of apoptosis, J. Pharmacol. Toxicol. Methods, 1997, 37, 215-228.

3. K. Saito, I. Wada, M. Tamura and M. Kinjo, Direct detection of caspase-3 activation in single live cells by cross-correlation analysis, Biochem. Biophys. Res. Commun., 2004, 324, 849-854.

4. $\quad$ R. Y. Tsien, The green fluorescent protein, Annu. Rev. Biochem., 1998, 67, 509-544.

5. M. Zimmer, Green fluorescent protein (GFP): Applications, structure, and related photophysical behavior, Chem. Rev., 2002, 102, 759-781.

6. Y. G. Li and M. S. Horwitz, Use of green fluorescent protein in studies of apoptosis of transfected cells, BioTechniques, 1997, 23, 1026-1029.

7. V. Shinbrot, C. M. Spencer and S. R. Kain, Morphological detection of plasma membrane changes during apoptosis using enhanced green fluorescent protein, BioTechniques, 1999, 26, 1064-1068.

8. H. S. Liu, M. S. Jan, C. K. Chou, P. H. Chen and N. J. Ke, Is green fluorescent protein toxic to the living cells?, Biochem. Biophys. Res. Commun., 1999, 260, 712-717.

9. K. J. Harvey, D. Lukovic and D. S. Ucker, Membrane-targeted green fluorescent protein reliably and uniquely marks cells through apoptotic death, Cytometry, 2001, 43, 273-278.

10. A. Strebel, T. Harr, F. Bachmann, M. Wernli and P. Erb, Green fluorescent protein as a novel tool to measure apoptosis and necrosis, Cytometry, 2001, 43, 126-133.

11. A. M. Steff, M. Fortin, C. Arguin and P. Hugo, Detection of a decrease in green fluorescent protein fluorescence for the monitoring of cell death: An assay amenable to high-throughput screening technologies, Cytometry, 2001, 45, 237-243.

12. P. I. H. Bastiaens and A. Squire, Fluorescence lifetime imaging microscopy: spatial resolution of biochemical processes in the cell, Trends Cell Biol., 1999, 9, 48-52.

13. H. Wallrabe and A. Periasamy, Imaging protein molecules using FRET and FLIM microscopy, Curr. Opin. Biotechnol., 2005, 16, 19-27.

14. K. Suhling, P. M. W. French and D. Phillips, Time-resolved fluorescence microscopy, Photochem. Photobiol. Sci., 2005, 4, 13-22.

15. J. R. Lakowicz, Principles of Fluorescence Spectroscopy, Springer, New York, third edn., 2006. 
16. W. Becker, A. Bergmann and C. Biskup, Multispectral fluorescence lifetime imaging by TCSPC, Microsc. Res. Tech., 2007, 70, 403-409.

17. H. P. Wang, T. Nakabayashi, K. Tsujimoto, S. Miyauchi, N. Kamo and N. Ohta, Fluorescence lifetime image of a single halobacterium, Chem. Phys. Lett., 2007, 442, 441-444.

18. T. Nakabayashi, H. P. Wang, M. Kinjo and N. Ohta, Application of fluorescence lifetime imaging of enhanced green fluorescent protein to intracellular $\mathrm{pH}$ measurements, Photochem. Photobiol. Sci., 2008, 7, 668-670.

19. T. Nakabayashi, I. Nagao, M. Kinjo, Y. Aoki, M. Tanaka and N. Ohta, Stress-induced environmental changes in a single cell as revealed by fluorescence lifetime imaging, Photochem. Photobiol. Sci., 2008, 7, 671-674.

20. M. Leist, B. Single, A. F. Castoldi, S. Kühnle and P. Nicotera, Intracellular adenosine triphosphate (ATP) concentration: a switch in the decision between apoptosis and necrosis, J. Exp. Med., 1997, 185, 1481-1486.

21. R. Pepperkok, A. Squire, S. Geley and P. I. H. Bastiaens, Simultaneous detection of multiple green fluorescent proteins in live cells by fluorescence lifetime imaging microscopy, Curr. Biol., 1999, 9, 269-272.

22. Q. S. Hanley, V. Subramaniam, D. J. Arndt-Jovin and T. M. Jovin, Fluorescence lifetime imaging: Multi-point calibration, minimum resolvable differences, and artifact suppression, Cytometry, 2001, 43, 248-260.

23. S. T. Hess, E. D. Sheets, A. Wagenknecht-Wiesner and A. A. Heikal, Quantitative analysis of the fluorescence properties of intrinsically fluorescent proteins in living cells, Biophys. J., 2003, 85, 2566-2580.

24. A. A. Heikal, S. T. Hess and W. W. Webb, Multiphoton molecular spectroscopy and excited-state dynamics of enhanced green fluorescent protein (EGFP): acid-base specificity, Chem. Phys., 2001, 274, 37-55.

25. M. A. Elsliger, R. M. Wachter, G. T. Hanson, K. Kallio and S. J. Remington, Structural and spectral response of green fluorescent protein variants to changes in $\mathrm{pH}$, Biochemistry, 1999, 38, 5296-5301.

26. R. M. Wachter and S. J. Remington, Sensitivity of the yellow variant of green fluorescent protein to halides and nitrate, Curr. Biol., 1999, 9, R628-R629.

27. G. H. Patterson and J. Lippincott-Schwartz, A photoactivatable GFP for selective photolabeling of proteins and cells, Science, 2002, 297, 1873-1877.

28. S. Matsuyama, J. Llopis, Q. L. Deveraux, R. Y. Tsien and J. C. Reed, Changes in intramitochondrial and cytosolic $\mathrm{pH}$ : early events that modulate caspase activation during apoptosis, Nat. Cell Biol., 2000, 2, 318-325. 
29. C. Nilsson, U. Johansson, A. C. Johansson, K. Kagedal and K. Ollinger, Cytosolic acidification and lysosomal alkalinization during TNF-alpha induced apoptosis in U937 cells, Apoptosis, 2006, 11, 1149-1159.

30. M. Cotlet, J. Hofkens, M. Maus, T. Gensch, M. Van der Auweraer, J. Michiels, G. Dirix, M. Van Guyse, J. Vanderleyden, A. J. W. G. Visser and F. C. De Schryver, Excited-state dynamics in the enhanced green fluorescent protein mutant probed by picosecond time-resolved single photon counting spectroscopy, J. Phys. Chem. B, 2001, 105, 4999-5006.

31. H. M. Shen and S. Pervaiz, TNF receptor superfamily-induced cell death: redox-dependent execution, Faseb J., 2006, 20, 1589-1598.

32. S. P. Yu, L. M. T. Canzoniero and D. W. Choi, Ion homeostasis and apoptosis, Curr. Opin. Cell Biol., 2001, 13, 405-411.

33. X. Li, T. Uchimura, S. Kawanabe and T. Imasaka, Use of a fluorescence lifetime imaging microscope in an apoptosis assay of Ewing's sarcoma cells with a vital fluorescent probe, Anal. Biochem., 2007, 367, 219-224.

34. T. Nakabayashi, M. Kinjo and N. Ohta, Electric field effects on fluorescence of the green fluorescent protein, Chem. Phys. Lett., 2008, 457, 408-412. 

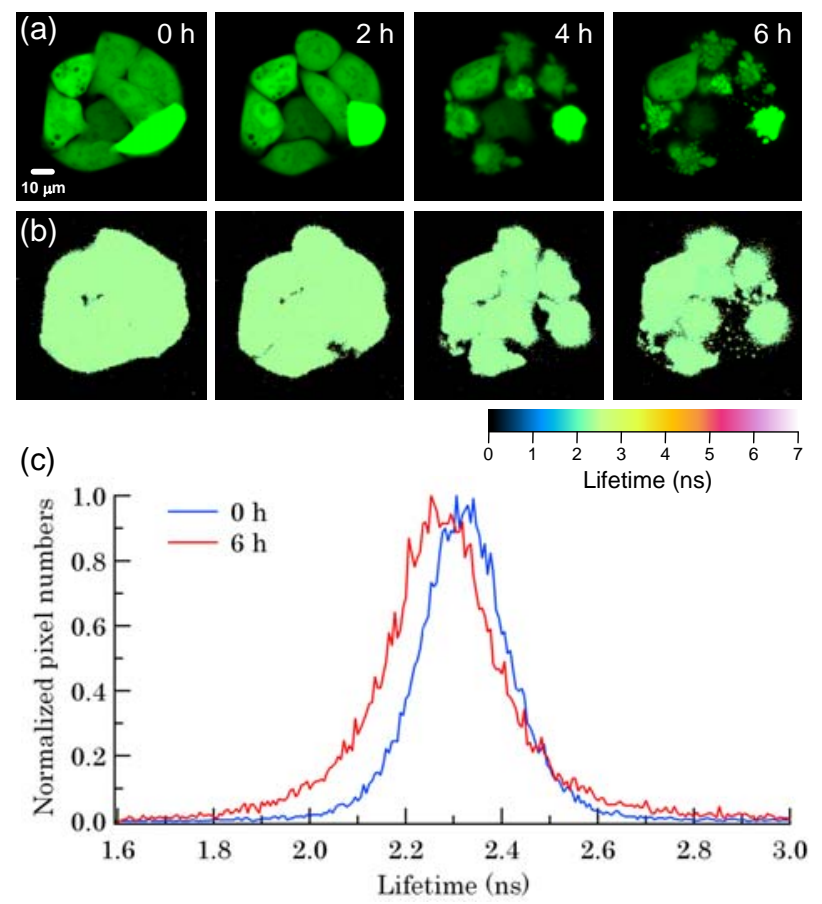

Fig. 1 Time course of the fluorescence intensity image (a) and fluorescence lifetime image (b) of HeLa cells expressing EGFP, after the apoptosis inducer was added, and the histograms of the lifetime distribution (c). Apoptosis was induced by $250 \mathrm{ng} / \mathrm{ml}$ TNF- $\alpha$ in combination with $14 \mu \mathrm{g} / \mathrm{ml} \mathrm{CHX.} \mathrm{Excitation}$ and monitoring wavelengths were 440 and 515-560 nm, respectively. Scale bar, $10 \mu \mathrm{m}$. 


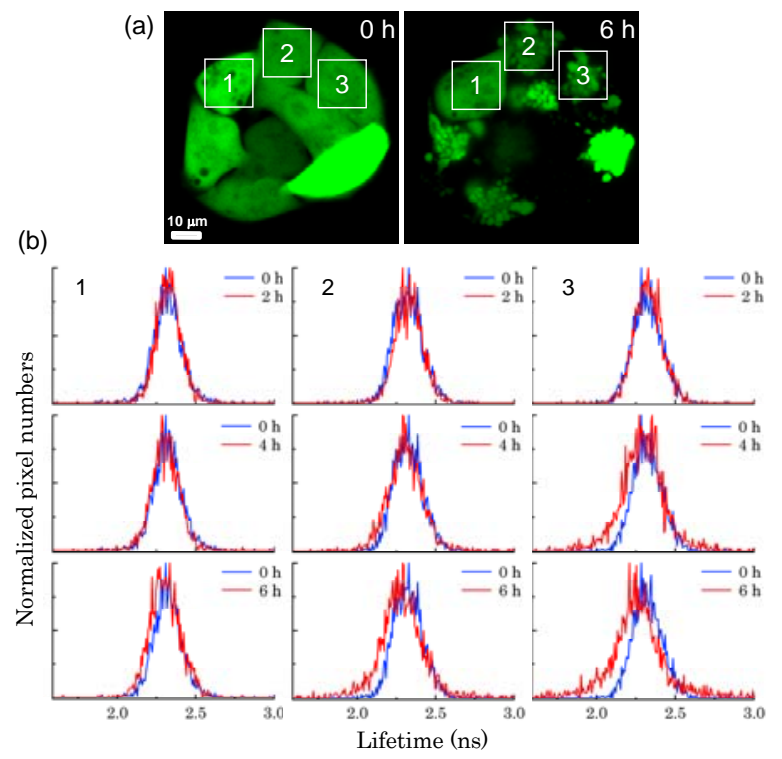

Fig. 2 (a) Fluorescence intensity images of HeLa cells expressing EGFP at 0 and $6 \mathrm{~h}$ after the apoptosis inducer was added. (b) Time course of the histogram of the fluorescence lifetime in different cells at 0, 2, 4 and $6 \mathrm{~h}$ after the apoptosis inducer was added. Excitation and monitoring wavelengths were 440 and 515-560 nm, respectively. Scale bar, $10 \mu \mathrm{m}$. 


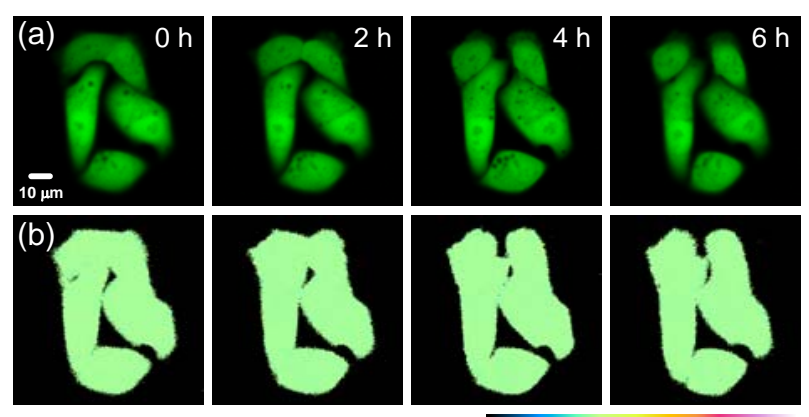

(c)
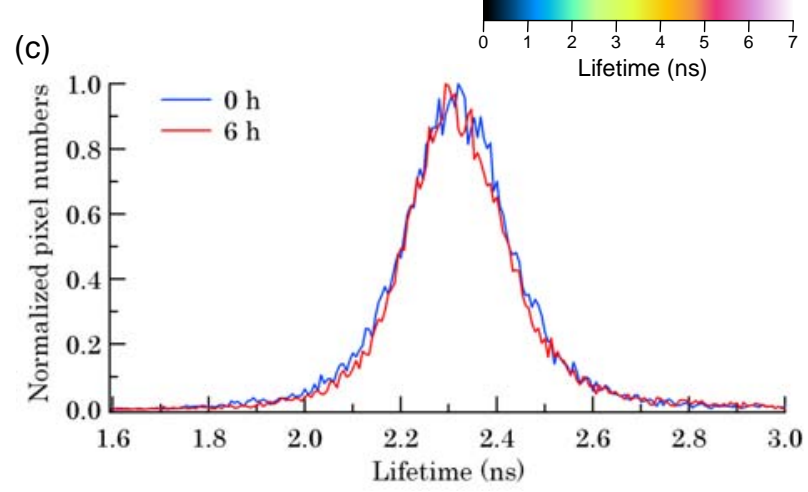

Fig. 3 Time course of fluorescence intensity image (a) and fluorescence lifetime image (b) of HeLa cells expressing EGFP observed without adding apoptosis inducer, and histograms of the lifetime distribution (c). Excitation and monitoring wavelengths were 440 and 515-560 nm, respectively. Scale bar is $10 \mu \mathrm{m}$. 

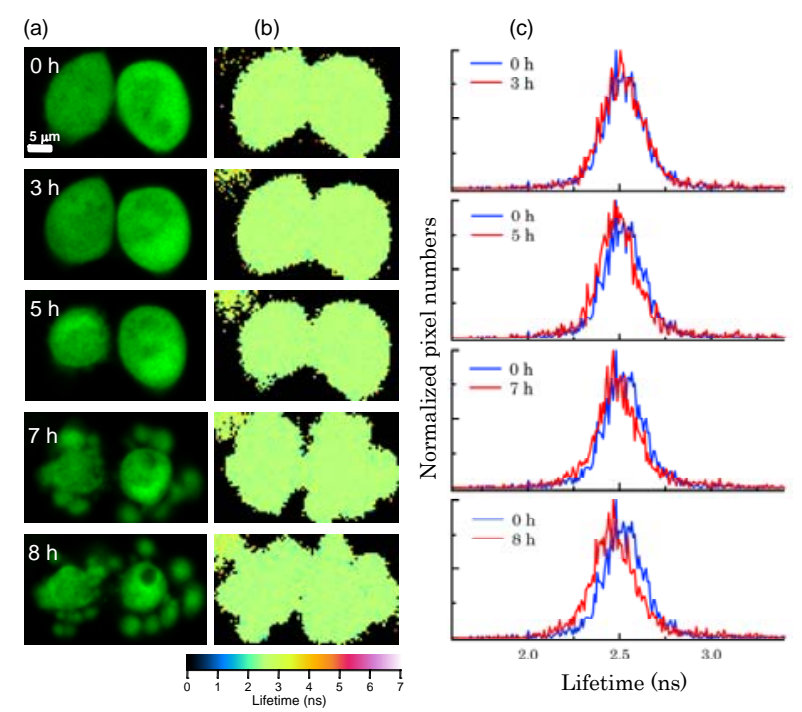

Fig. 4 Time course of fluorescence intensity image (a), fluorescence lifetime image (b), and the fluorescence lifetime distribution (c) of HeLa cells expressing EGFP, after the apoptosis inducer was added. Excitation and monitoring wavelengths were 405 and $509 \pm 5 \mathrm{~nm}$, respectively. Scale bar is 5 $\mu \mathrm{m}$. 

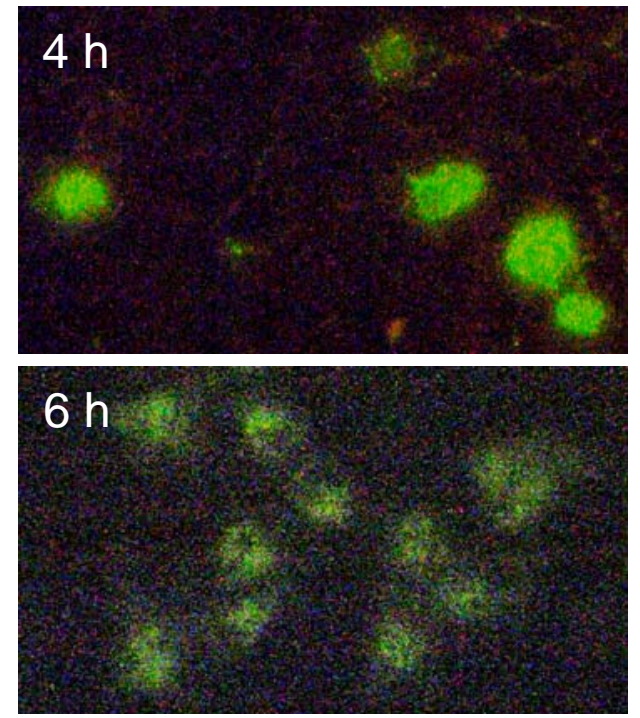

Fig. 5 Representative fluorescence images of annexin V-FITC staining of apoptosis in HeLa cells at 4 and 6 hours after apoptosis induction. Apoptosis was induced by $250 \mathrm{ng} / \mathrm{ml}$ TNF- $a$ in combination with $14 \mathrm{mg} / \mathrm{ml}$ CHX. 